\title{
Quinua, Chenopodium quinua (Willd.) en Colombia Caracterización de granulos de almidón nativo de quinua por IR-ATR, MEB, DRX
}

pags $122-131$

Grupo de Investigación: Centro de Bioprospección e Ingeniería Química Aplicada al Estudio de

Biomoleculas e Industria -BIQA

Línea de investigación: Almidones Funcionalizados

Claudio Raúl Bernal Bustos` Luisa Fernanda Ramírezº, Paula Daniela Duarte», Ana María Guzmán»* \& Juliana Acero•

\section{RESUMEN}

El cultivo de quinua es una alternativa productiva que empieza a dimensionarse-rentable en Colombia, se extiende sin control en los departamentos de Nariño, Cauca, Boyacá, Cundinamarca, parte alta del Departamento del Putumayo y Antioquia, lo anterior, a razón de las bondades que posee el grano teniendo en cuenta sus propiedades nutricionales y saludables, asi como del valor comercial que representa en el entorno internacional. En este artículo se estudia los gránulos de almidón contenido en granos-semillas de quinua que se cultivan en Colombia, además se trata de proveer información de las características del almidón mediante IR-ATR, MEB y DRX, posibilitando dos aspectos, por un lado, definir el potencial de uso del almidón en diferentes industrias, e igualmente, aspectos de cultivo, cosecha, poscosecha y comercialización de la quinua que se expende en Colombia.

Palabras Claves: Chenopdium quinua (Willd.), quinua, almidón, Infrarrojo, microscopía electrónica de barrido, difracción de rayos $X$.

\section{ABSTRACT}

The harvesting of quinoa is a productive alternative that starts to be-profitable in Colombia extends way excessive in the departments of Nariño, Cauca, Boyacá, Cundinamarca, upper part of the Department of Putumayo, Antioquia, above, at the rate of the goodness that has grain taking into account their nutritional and healthy properties as well as the commercial value that represents in the international environment. This article explores the starch granules contained in grain-seeds of quinoa grown in Colombia, it is also providing information on the characteristics of starch by ATR-IR, SEM and XRD, enabling two aspects, on the one hand, to define the potential of use of starch in different industries, and also aspects of cultivation, harvesting, post-harvest and commercialization of quinoa that is sold in Colombia

Key Words: Chenopdium quinoa (Willd.), starch, quinoa, electron microscopy, infrared scanning, $x$-ray diffraction.

\footnotetext{
- Conducente al grado científico de doctor en Avances en Ciencia y Biotecnología Alimentarias, Universidad de Burgos - Burgos, España, Investigador Fundación Universidad de América. claudio.bernal@investigadores.uamerica.edu.co

•- Estudiantes del programa de Ingeniería Química, Fundación Universidad de América.
} 
LINNEA DE INVESTIGACIÓN: ALMIDORES FUNCIONALIZADOS

\section{INTRODUCCIÓN}

El almidón es un hidrato de carbono que constituye la principal reserva energética de casi todos los vegetales. Se caracteriza por ser, y para objeto industrial, una materia prima económica y abundante, lo constituye una ventaja para su utilización futura. Sin embargo, las partículas de almidón presentan algunas limitaciones de uso en su estado nativo, lo que afecta su procesabilidad y aplicabilidad.

Las propiedades físico-químicas y funcionales del almidón y sus productos dependen de su naturaleza, morfología y estructura; características que se ven influenciadas y/o varían de acuerdo a las prácticas y latitudes de cultivo.

En Colombia se viene promoviendo el cultivo de quinua, aunque, como lo refiere Bernal et al., (2015), "la quinua en Colombia es un cultivo incipiente... la única perspectiva futura sigue modelos de producción agricola y de agroindustria propuesta por los países andinos productores $y$ comercializadores de grano y de alimentos con bajo valor agregado.

El grano-semilla de quinua es un compendio de posibilidades y oportunidades (C Bernal, 2015) 2015); (Prego, Maldonado, \& Otegui, 1998) define tres secciones, fundamentalmente el grano-semilla se encuentra conformado por embrión, endospermo y perisperma; (Ando et al., 2002) obtuvieron 38,3\% de salvado y embrión y $58,8 \%$ de perisperma. En este sentido, (Claudio Bernal, Cárdenas, Rozo, Daza, \& Echeverry, 2014) plantea un nuevo escenario para Colombia a partir de las fracciones constitutivas teniendo en cuenta la estructura y morfología del grano-semilla quinua, para lo cual propone dos fracciones primarias de partidaFPP, una de ellas, FPP1 $40-45 \%$, conformada por el conjunto de embrión, endospermo y otros tejidos localizados en el perisperma y la fracción FPP2 50-55\%, corresponde al perisperma. En FPP1 se encuentran localizados macro y micronutrientes: proteínas (Brinegar, Sine, \& Nwokocha, 1996; Ruales \& Nair, 1992), grasas
(Koziol, 1943; Ng \& Anderson, n.d.), vitaminas (xxx), minerales (Repo-Carrasco-Valencia, Encina, Binaghi, Greco, \& Ronayne de Ferrer, 2010), fitoconstituyentes (flavonas (Chlopicka, Pasko, Gorinstein, Jedryas, \& Zagrodzki, 2012; Dini, Carlo Tenore, \& Dini, 2004; Hirose, Fujita, Ishii, \& Ueno, 2010), fenoles (Chlopicka et al., 2012; Gorinstein et al., 2008; Miranda et al., 2010; P. Paśko, Sajewicz, Gorinstein, \& Zachwieja, 2008; Paweł Paśko et al., 2009), ecdisteroides (Foucault et al., 2012; Graf et al., 2014; Kumpun et al., 2011; Nsimba, Kikuzaki, \& Konishi, 2008; Zhu et al., 2001) y otros componentes como antinutrientes: fitatos, saponinas, inhibidores de tripsina y actividad de lipoxigenasa (Ando et al., 2002). La fracción FPP2 y/o perisperma, en su conjunto, puede separase del resto del grano (cotiledones, embrión, endospermo). Este protocolo experimental fue establecido como Imbibición Inducida-II01UBU y desarrollado en el laboratorio de Tecnología de los Alimentos, Grupo Tecnofoods - Facultad de Ciencias, Universidad de Burgos (España). El proceso de separación del almidón del perisperma, a escala de laboratorio, fue realizado por el Grupo BIQA en los laboratorios de Ingeniería Química de la Universidad de América (Colombia); empleando un proceso de decantación y filtración inducida - Método II01FUA. Los métodos antes mencionados no hacen parte de este informe.

El presente trabajo tiene como propósito caracterizar el almidón nativo de quinua contenido en grano-semillas de variedades que se cultivan en Colombia y adquiridas bajo condiciones ex situ, procedentes de los principales centros de producción y localizados en los Departamentos de Nariño, Cauca, Cundinamarca y Boyacá. De hecho, en Colombia, la agricultura de la quinua aún no está muy desarrollada, sus prácticas agronómicas son sin importancia, no existe mecanización, no se dispone de semillas, sin químicos como pesticidas y herbicidas. Lo anterior, y en el marco de constituir una cadena productiva y de valor de la quinua en Colombia, es hora de empezar a definir escenarios a corto, mediano y largo plazo para el cultivo y la agroindustria de productos con un alto valor agregado, 
dado que la caracterización del almidón, gránulos, nos permite encontrar indicios acerca de las prácticas y lugares donde se llevan cabo los cultivos de quinua.

\section{MATERIALES Y MÉTODOS}

\section{Ubicación del área de acceso en condiciones ex situ}

El almidón se obtiene a partir de granossemillas de Chenopodium quinua (Willd.) procedentes de mercados localizados en la ciudades de Ipiales y Pasto (Nariño), Popayán (Cauca), Soracá, Tunja, Duitama (Boyacá), Tabio y la Calera; en Cundinamarca.

En este artículo, y como una primera aproximación a la definición del estado de arte en el tema de quinua en Colombia, las muestras fueron agrupadas en tres (3) zonas: Z1_Nariño, Z2_Cauca y Z3_Boyacá. Las muestras que corresponde al Departamento de Cundinamarca (Z4) no hacen parte de este informe.

\section{Extracción del almidón de muestras de granos-semillas de quinua}

Para la extracción del almidón fue empleado el método II01UBU; protocolo experimental que permite forzar un rompimiento del funículo, dejando un grano "desmembrado-abierto. A continuación se realiza una molienda húmeda del producto, se pasa a un decantador centrífugo que permite obtener dos fracciones primarias de partida - FPP1 y 2. La fracción FPP1 corresponde al conjunto de tejidos conformados por el embrión, endospermo (Prego et al., 1998) y otros tejidos presentes en el perisperma; que actúan como "bolsas" los cuales contienen los gránulos de almidón. Finalmente FPP1 se estabiliza la humedad a $70^{\circ} \mathrm{C}$ por 1 hora, se ajusta el tamaño de partícula y se empaca.

La fracción FPP2 se somete a una reacción alcalina con $\mathrm{NaOH}(5-7 \%)$, se agita y se somete a una decantación forzada hasta obtener dos fases. Seguidamente se elimina el sobrenadante y se realizan dos (2) lavados más uno (1), los dos primeros lavados se realiza con agua a temperatura entre $0-5^{\circ} \mathrm{C}$ hasta obtener el crudo de almidón, se finaliza con un tercer lavado del crudo de almidón con agua atemperada a 40$45^{\circ} \mathrm{C}$. El crudo de almidón se seca en una cámara de Oven TR 60-TR1050 Nabertherm, a una temperatura por debajo de la temperatura de gelatinización $<55^{\circ} \mathrm{C}$, durante 24 horas, el producto-escamas lleva a un molino de bolas CENMKII-12 y se finaliza con un ajuste del tamaño de partícula en CEN-MKII-13.

\section{Ensayos fisicoquímicos}

Humedad, técnica usada AOAC Internacional 925.19/05; cenizas, AOAC Internacional 940.26/05; Proteínas, AOAC Internacional 960.52; Grasa, AOAC Internacional 960.39/95; Fibra cruda, AOAC Internacional 962.09/05; Carbohidratos reductores, Método ácido dinitrosalicilico (DNS); Carbohidratos totales, cálculo por diferencia y Aporte Calórico: Método numérico.

\section{Ensayos microbiológicos}

Coliformes totales: AOAC 991.15; Coliformes fecales: AOAC 992.30; Hongos y levaduras: NTC4132; Mesófilos: AOAC 990.12.

\section{IR-ATR}

Thermo Scientific Nicolet iS10 con un módulo de ATR (por sus siglas en inglés: Attenuated Total Reflection) verificado con patrones de Poliestireno $1.5 \mathrm{~mm}$ trazables a los estándares NIST (por sus siglas en inglés: National Institute of Standards and Technology).

\section{MEB}

Este análisis se usa para examinar la superficie y morfología de los gránulos de almidón nativo. Para este análisis se usó un equipo SEM Oxford 7359 - Metalización en oro y observación en alto vacío, con aumentos de 10000X. 
LIINEA DE INVESTIGACIÓN: ALMIDORES FUNCIONALIZADOS

\section{DRX}

La obtención de patrón de Difracción de Rayos X, Patrón de cristalinidad del almidón nativo de quinua se llevó a cabo en un equipo Rigaku Ultima III. Las condiciones de operación del ensayo consistieron en una maceración de la muestra, a una Temperatura de $23^{\circ} \mathrm{C}$ y una humedad relativa del $50 \%$.

\section{RESULTADOS}

Análisis proximal de almidón nativo de quinua, muestras Z1 (Nariño), Z2 (Cauca) y Z3 (Boyacá).

En la Tabla 1 se muestra la composición del almidón nativo de quinua para Z1, Z2 y Z3 y de acuerdo al método II01FUA. Los ensayos microbiológicos no reportan la presencia de coliformes hongos y levaduras.

Tabla 1. Composición del almidón de quinua para las Zonas Productoras estudiadas.

\begin{tabular}{|c|c|c|c|c|c|c|c|c|c|}
\hline & $\begin{array}{l}\text { Humedad } \\
\text { (\%) }\end{array}$ & $\begin{array}{c}\text { Materia } \\
\text { seca } \\
(\%)\end{array}$ & $\begin{array}{c}\text { Proteínas } \\
\text { (\%) }\end{array}$ & $\begin{array}{c}\text { Grasa } \\
(\%)\end{array}$ & $\begin{array}{c}\text { Fibra } \\
\text { cruda } \\
(\%)\end{array}$ & $\begin{array}{c}\text { Carbohidratos } \\
\text { (\%) }\end{array}$ & $\begin{array}{l}\text { Carbohidratos } \\
\text { redutores (\%) }\end{array}$ & $\begin{array}{c}\text { Cenizas } \\
(\%)\end{array}$ & $\begin{array}{l}\text { Energía } \\
\text { bruta (\%) }\end{array}$ \\
\hline Z1 & 9.02 & 86.03 & 2.04 & 1.92 & 3.60 & 72.00 & 21.07 & 0.39 & $4,466 \cdot 10$ \\
\hline $\mathrm{Z} 2$ & 12.07 & 87.93 & 4.80 & 3.57 & 3.90 & 75.17 & 22.75 & 0.49 & $4,566.80$ \\
\hline Z3 & 12.01 & 85.04 & 4.01 & 3.23 & 3.01 & 73.20 & 22.02 & 0.41 & $4,500.02$ \\
\hline
\end{tabular}

Z1: Nariño; Z2: Cauca; Z3: Boyacá. Los valores: Humedad, técnica usada AOAC Internacional 925.19/05; Cénizas, AOAC Internacional 940.26/05; Proteínas, AOAC Internacional 960.52; Grasa, AOAC Internacional 960.39/95; Fibra cruda, AOAC Internacional 962.09/05; Carbohidratos reductores, Método ácido dinitrosalicilico (DNS); Carbohidratos totales, cálculo por diferencia y Aporte Calórico: Método numérico. Los valores que se presentan en la misma columna son significativamente diferentes $(p<05)$.

Fuente: Autores

Análisis por IR-ATR

La espectroscopia IR-ATR se utilizó para evaluar el espectro de la sustancia extraída del granosemilla de quinua, correspondiendo a un almidón. En la Tabla 1 y Gráficas 1 a 3 se muestran los datos de los almidones de las muestras de tres (3) zonas productoras colombianas. El análisis de espectroscopia infrarroja evidencia que el almidón nativo presenta vibraciones en el rango de 1300 a $1500 \mathrm{~cm}^{-1}$ típicas para los almidones. La interpretación de los análisis por FT-IR de los almidones, según(Kizil, Irudayaraj, \& Seetharaman, 2002), se debe llevar a cabo en 4 principales regiones, que permite la caracterización de las bandas claves. Estas regiones son: Por debajo de $800 \mathrm{~cm}^{-1}$, entre $800-1500 \mathrm{~cm}^{-1}$ (región dactilar), la región entre 2800 y $3000 \mathrm{~cm}^{-1}$ (región del estiramiento $\mathrm{C}-\mathrm{H}$ ) y la región entre 3000 y $3600 \mathrm{~cm}^{-1}$ (denominada como la zona del estiramiento $\mathrm{OH}$ ).
Z1 está por debajo de $800 \mathrm{~cm}^{-1}$ se presentan vibraciones complejas a causa del esqueleto del anillo de la glucosa, en la segunda se encuentran solapamientos de espectros complejos, por lo que la asignación se hace difícil; en los almidones estos espectros se origina por la vibración de las unidades monoméricas de glucosa y esto ha hecho que este modo de vibración se le asigne a los almidones, por lo que en esta región los almidones exhiben características espectrales muy similares. En los resultados obtenidos las bandas de 928,$79 ; 928,72$ y $928,72 \mathrm{~cm}^{-1}$ para los almidones nativos estudiados son atribuidos a los enlace glucosídico a 1-4 (C-O-C) de la amilosa presente en los almidones, las vibraciones en el rango de 1300 a $1500 \mathrm{~cm}^{-1}$ representan la flexión o deformación de los carbonos e hidrógenos. El agua absorbida en la región amorfa se identifica en las bandas de 1644,09; 1639,09 y $1643,62 \mathrm{~cm}^{-1} \mathrm{y}$ hacen referencia a la cristalinidad 
de los almidones, la cual varía según el origen y tipo de almidón, lo que indica que a pesar de ser extraído de diferentes semillas de quinua $Z 1, Z 2$ y Z3, presentan similitudes - Tabla 2.

\section{Gräficas IR}

Gráfica 1. Muestra Nariño - Z1

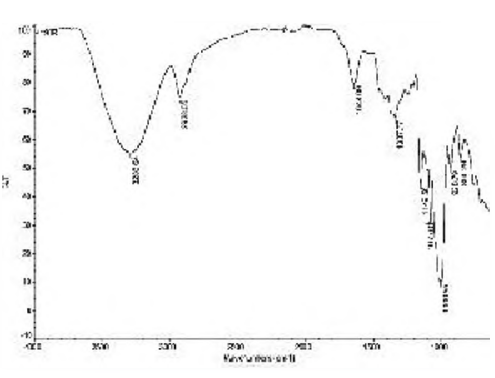

Gráfica 2. Muestra Cauca - Z2

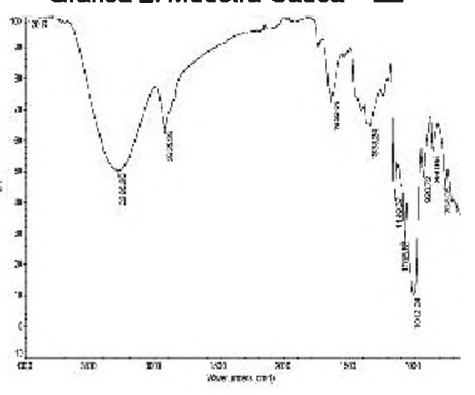

Gráfica 3. Muestra Boyacá - Z3

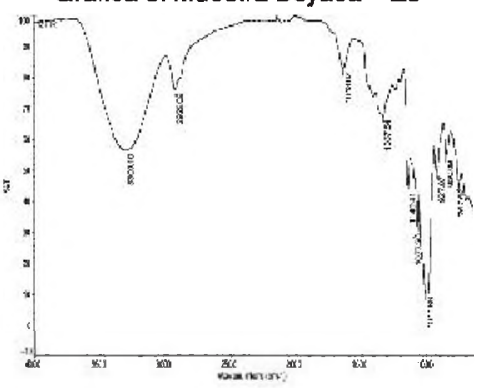

Fuente: Autores

Tabla 2. Resultados de IR

\begin{tabular}{|c|c|c|l|}
\hline \multicolumn{3}{|c}{ No. de onda (Cm-1) } \\
\hline \multirow{2}{*}{$z$} & 763.05 & 762.62 & \multicolumn{2}{c|}{ Astignacón banda infrarroja } \\
\hline 860.29 & 859.65 & 860.09 & Deformación C-H y $\mathrm{CH}_{2}$ \\
\hline 928.79 & 928.72 & 927.87 & Vibración del enlace glucosídico $\alpha$ 1-4 (C-O-C) \\
\hline 1000.55 & 1012.24 & 1011.86 & C(1)-H Flexión \\
\hline 1077.01 & 1076.88 & 1077.20 & C-O-H flexión \\
\hline 1149.56 & 1149.37 & 1149.41 & C-O, C-C estiramiento \\
\hline$*$ & $*$ & $*$ & Deformación de la cadena lateral $\mathrm{CH}_{2} \mathrm{OH}$ \\
\hline 1337.77 & 1338.24 & 1337.54 & Flexión de C-O-H, torsión de $\mathrm{CH}_{2}$ \\
\hline$*$ & $*$ & $*$ & Flexion de $\mathrm{CH}_{2}$ \\
\hline 1644.09 & 1639.09 & 1643.62 & Agua adsorbida de la región amorla \\
\hline 2928.05 & 2925.29 & 2929.02 & Deformación $\mathrm{CH}_{2}$ \\
\hline 3286.64 & 3285.20 & 3300.10 & Estiramiento de $\mathrm{OH}$ \\
\hline
\end{tabular}

Fuente: Autores

\section{Morfología mediante Microscopía Electrónica de Barrido}

Las imágenes obtenidas para los gránulos de almidón, mediante una microscopia de barrido para el almidón nativo de quinua, muestran que los gránulos presentan una forma poligonal que corresponde a la reportada en
(Lindeboom, Chang, Falk, \& Tyler, 2005) y un tamaño de granulo de aproximadamente 1,22 $\mu m$, la cual se puede observar en la figura 1 , que coincide en el rango de 1 - $2 \mu \mathrm{m}$ reportados por (Ando et al., 2002; Atwell, Patrick, Johnson, \& Glass, 1983; Claudio Bernal et al., 2014; Lindeboom et al., 2005; Tang, Watanabe, \& Mitsunaga, 2002). 
Figura 1. Morfologla mediante Microscopla Electrónlca de Barrldo de Almldón natlvo de quinua; tres (3) Zonas

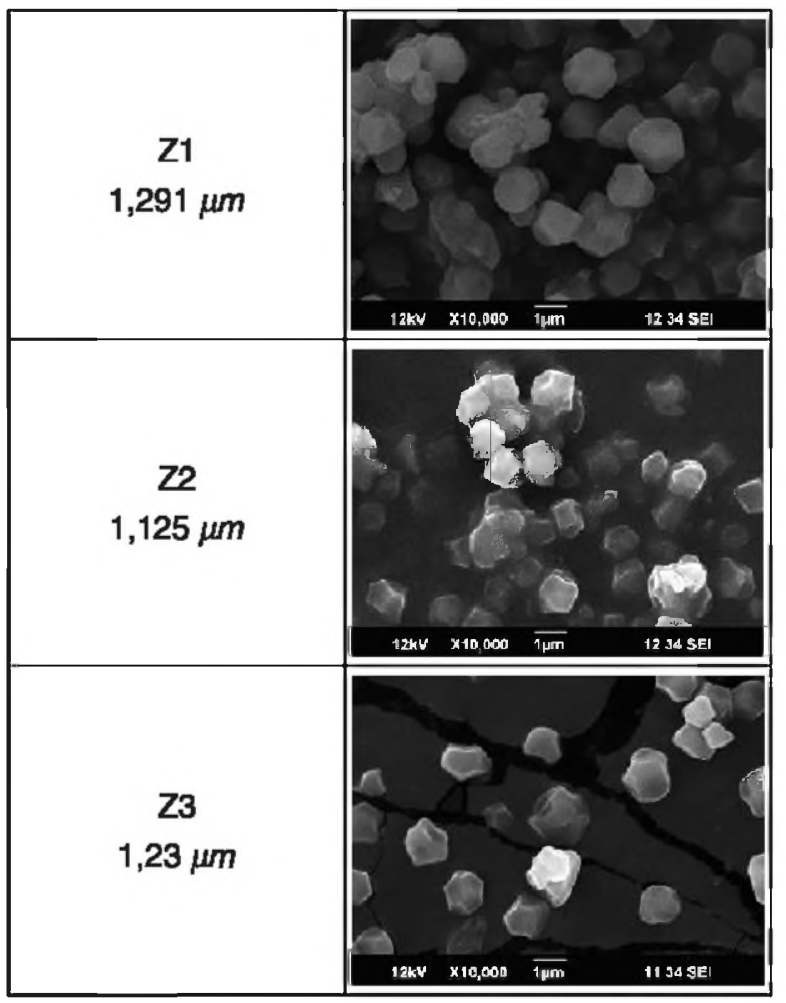

Fuente: Autores

\section{Patrón de Difracción de Rayos $X$, patrón de Cristalinidad del almidón nativo de quinua}

La Gráfica 4 muestra el difractograma correspondiente al almidón nativo de quinua. Los valores encontrados fueron: Área cristalina $=45300$, área total=280036, porcentaje de cristalinidad $(\%)=16,176$.

\section{DISCUSIÓN}

\section{Extracción de almidón}

Múltiples métodos se han empleado para la separación del almidón a partir de granos-semillas de quinua. Los métodos más usuales han sido vía molienda-húmeda, previo remojo de los granos-semillas con tampón de acetato a pH 6.5 (Atwell et al., 1983; C Bernal, Leal, \& Garzón, 2009; Claudio Bernal, Daza, \& Echeverry, 2011; Escobar, De la Pava, \& Bemal, n.d.; Pastás, Pineda, \& Bernal, 2012) usando el mismo método, pero previo remojo con $0.3 \%(\mathrm{p} / \mathrm{v}) \mathrm{de}$ $\mathrm{NaOH}$. (Pe, Steffolani, \& Leo, 2013) evaluó dos procesos básicos reportados por (Wright, Huber, Fairbanks, \& Huber, 2002), donde para la extracción parte de harina de quinua la cual fue suspendida en una solución de $\mathrm{NaOH} 0.25 \%$, se agita y finalmente se centrifuga por $20 \mathrm{mi}-$ nutos a $2465 \times$ g. Este autor menciona que la molienda en seco, a pesar de ser un método más rápido, éste causa daño en gránulos, por su parte las condiciones alcalinas, en etapas de remojo del grano, cambian las propiedades del almidón y que no es posible ser usado este almidón para fines termoplásticos. (Lindeboom et al., 2005) emplea un método húmedo a partir de semillas de quinua, usando agua desionizada, luego se pasa a molino, se filtra y finalmente se centrifuga a $4300 \times \mathrm{g}$.

\section{Gráfica 4. Difractograma de almidón nativo de} quinua

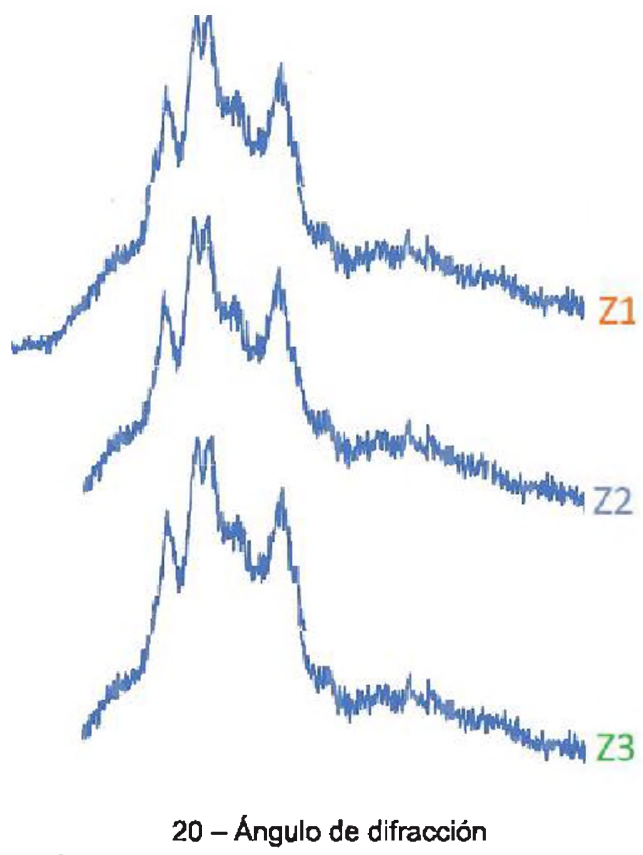

Fuente: Autores 


\section{Composición del almidón}

La composición del almidón aislado de quinua, en las tres zonas estudiadas se presenta en la Tabla 1. Mientras (Pe et al., 2013), para las variedades de quinua estudiadas, base seca, presentan una humedad promedio de $10.04 \%$, proteína de $1.11 \%$ y $1.23 \%$ (Lindeboom et al., $2005)$, grasa de $2.16 \%$ y cenizas de $1.53 \%$, la composición del almidón de variedades colombianas señalan $11.03 \%$; $3.62 \%$, $2.91 \%$ y $0.43 \%$ humedad, proteína, grasa y cenizas, respectivamente, encontrando una gran diferencia que, en principio, puede deberse al método de extracción empleado.

MEB

El tamaño, forma y estructura de los gránulos de almidón difieren sustancialmente de la fuente botánica). La Figura 1 muestra los gránulos de almidón mediante Microscopía Electrónica de Barrido - MEB para las tres zonas estudiadas. Mientras la quinua es un caso extremo de agregados granulares, que se agrupan en con más de 100 gránulos (Atwell et al., 1983), en este estudio se pudo confirmar agregados de aproximadamente $25 \mu \mathrm{m}$ y un tamaño granular entre $1.12 \mu \mathrm{m}$ a $1.23 \mu \mathrm{m}$. (Claudio Bernal et al., 2014) para muestras de Nariño - Mercado de Pasto determinaron un tamaño de gránulos entre $1.3 \mathrm{y}$ $1.8 \mu \mathrm{m}$, a diferencia de lo que reporta (Pe et al., 2013) quiénes encontraron gránulos entre un rango de 1 a $3.5 \mu \mathrm{m}$ y un promedio de $2.53 \mu \mathrm{m}$;

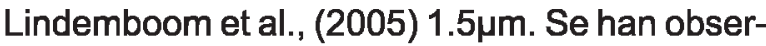
vado agregados entre 18 y $20 \mu \mathrm{m}$. No obstante, en todos los casos, la forma de los gránulos de almidón de quinua es forma poligonal irregular.

\section{IR-ATR}

La interpretación de los análisis por FT-IR de los almidones, según (Kizil et al., 2002), se debe llevar a cabo en 4 principales regiones, que permite la caracterización de las bandas claves. Estas regiones son: Por debajo de $800 \mathrm{~cm}^{-1}$, entre $800-1500 \mathrm{~cm}^{-1}$ (región dactilar), la región entre 2800 y $3000 \mathrm{~cm}^{-1}$ (región del estiramiento $\mathrm{C}-\mathrm{H}$ ) y la región entre 3000 y $3600 \mathrm{~cm}^{-1}$ (denominada como la zona del estiramiento $\mathrm{OH}$ ).

En la primera zona por debajo de $800 \mathrm{~cm}^{-1}$ se presentan vibraciones complejas a causa del esqueleto del anillo de la glucosa, en la segunda se encuentran solapamientos de espectros complejos, por lo que la asignación se hace difícil; en los almidones estos espectros se origina por la vibración de las unidades monoméricas de glucosa y esto ha hecho que este modo de vibración se le asigne a los almidones, por lo que en esta región los almidones exhiben características espectrales muy similares. En los resultados obtenidos las bandas de 928,79; 928,72 y $928,72 \mathrm{~cm}^{-1}$ para los almidones nativos estudiados son atribuidos a los enlace glucosídico $\alpha 1-4$ (C-O-C) de la amilosa presente en los almidones, las vibraciones en el rango de 1300 a $1500 \mathrm{~cm}^{-1}$ representan la flexión o deformación de los carbonos e hidrógenos. El agua absorbida en la región amorfa se identifica en las bandas de 1644,$09 ; 1639,09$ y $1643,62 \mathrm{~cm}^{-1}$ y hacen referencia a la cristalinidad de los almidones, la cual varía según el origen y tipo de almidón, lo que indica que a pesar de ser extraído de diferentes semillas de quinua $A 1, A 2$ y $A 3$, presentan similitudes.

\section{$\mathrm{DRX}$}

La Gráfica 4 muestra los difractogramas y los grados de cristalinidad del almidón nativo de quinua para Z1 (Nariño), Z2 (Cauca) y Z3 (Boyacá). Los gránulos de almidón de quinua presentaron un patrón de difracción Tipo $\mathrm{A}$, siendo los picos ubicados en $2 \theta=14.95,17.1,18.1$, 23.05 los de mayor intensidad, similares a los indicados en un patrón de DRX para almidones nativos.

Los valores de cristalinidad para las muestras analizadas de almidón de quinua fueron de $16.176 \%$, estando dentro del valor de $15 \%$ a 45\% como lo indica (Taylor, 2009: Zobel HF. Starch/Stärke. 1988; 40:40), pero difiere a los indicados por (Pe et al., 2013) en las variedades analizadas encontrando, en promedio de 3 
muestras, un valor de $37.9 \%$; (Tang et al., 2002) $35.0 \%$. La diferencia podría deberse a que los autores emplean muestras, para el caso de $(\mathrm{Pe}$ et al., 2013). éstas son suministradas por PROIMPA; banco de germoplasma de Bolivia y, por parte de (Tang et al., 2002), utilizó semillas de quinua de la variedad Real, Chenopodiaceae; Perú. En contraste, en la presente investigación se emplearon granos de quinua adquiridas bajo condiciones ex situ, en plazas de mercado, tiendas, etc. Una de las muestras estudiada por (Pe et al., 2013) es la Q-Kurmi obtenida desde la variedad amarilla de maranganí muestra una grado de cristalinidad de $39.6 \%$. La muestra de Boyacá, referenciada como Z2, corresponde a una variedad que se cultiva en Boyacá; granos de color amarillo y el cual fue reportado por el proveedor como amarilla de maranganí, pero se desconoce su origen, prácticas de cultivo, etapas de poscosecha, etc., cuyo grado de cristalinidad determinado fue de $15.99 \%$, lo que indica una diferencia muy marcada entre éstas. Esto puede deberse a las prácticas de cultivo, la zonacultivos y, quizá por otro lado, a los métodos de extracción del almidón, dado que los valores del contenido de proteína que hemos encontrado es de 4.01 en contraste a $1.09,0.565,0.30$ reportadas por (Lindeboom et al., 2005; Pe et al., 2013); respectivamente.

\section{CONCLUSIONES}

El nuevo enfoque de negocio y agroindustria de la quinua en Colombia y concebido por Bernal et al., 2015, a partir de fraccionar el grano-semilla, no diferencia variedades de quinua respecto a si son de variedad dulce y/o quinua amarga. Este enfoque permite utilizar el $100 \%$ de la cosecha y evita consideraciones de procesos como el proceso de desaponificación y/o consecuencias colaterales ambientales y económicas.

Las condiciones asociadas a las zonas de cultivo, a sus prácticas, al tipo de semilla, etapas de poscosecha, almacenamiento, procesos de limpieza de saponinas, empaques, etc., podrían ser los factores que se ven inmersos en la calidad del producto, dado que en Colombia, como ya se ha expresado, la quinua es un cultivo incipiente. De hecho, en Colombia, la agricultura de la quinua aún no está muy desarrollada, sus prácticas agronómicas son sin importancia, no existe mecanización, no se dispone de semillas, no se han definido los químicos como pesticidas $y$ herbicidas.

\section{REFERENCIAS}

Ando, $H_{\text {., }}$ Chen, Y., Tang, H., Shimizu, M., Watanabe, K., \& Mitsunaga, T. (2002). Food Components in Fractions of Quinoa Seed. Food Sci. Tecnol. Res., 8(1), 80-84.

Atwell, W., Patrick, B., Johnson, L., \& Glass, R. (1983). Characterization of quinoa starch.pdf. Cereal Chemistry, 6O(1), 9-11.

Bernal, C. (2015). Quinua (Chenopodium quinoa Willd) en Colombia . Primera, (164), 1-31.

Bernal, C., Cárdenas, O., Rozo, W., Daza, P., \& Echeverry, A. M. (2014). Octenilsuccinato alumínico de almidón de quinua - grado cosmético- $y$ su certificación bajo la denominación de ingrediente natural. Revista de Investigación Fundación Universidad de América, 7, 38-49. Retrieved from www.uamerica.edu.oc

Bernal, C., Daza, P., \& Echeverry, A. M. (2011). Certificación Internacional de Productos de Investigación (Nuevo conocimiento). Reciteia, 11(1a), 23. Retrieved from https://docs. google.com/file/d/0B476 jmP8wnvhbjdOdmlrb2lyNUE/edit?pli=1

Bernal, C., Leal, A., \& Garzón, J. (2009). Obtención a escala laboratorio del octenilsuccinato alumínico de almidón de quinua con miras a su utilización en un producto cosmético. Virtual Pro, 2008. Retrieved from http://revistavirtualpro. com/files/T101_200912.pdf

Brinegar, C., Sine, B., \& Nwokocha, L. (1996). High- 
Cysteine 2S Seed Storage Proteins from Quinoa ( Chenopodium quinoa ). Journal of Agricultural and Food Chemistry, 44(7), 1621-1623. doi:10.1021/jf950830+

Chlopicka, J., Pasko, P., Gorinstein, S., Jedryas, A., \& Zagrodzki, P. (2012). Total phenolic and total flavonoid content, antioxidant activity and sensory evaluation of pseudocereal breads. LWT - Food Science and Technology, 46(2), 548-555. doi:10.1016/j. Iwt.2011.11.009

Dini, I., Carlo Tenore, G., \& Dini, A. (2004). Phenolic constituents of Kancolla seeds. Food Chemistry, 84(2), 163-168. doi:10.1016/S03088146(03)00185-7

Escobar, J., De la Pava, S., \& Bernal, C. (n.d.). Diseño de una máquina limpiadora y clasificadora, por dimensiones, de granos-semillas de Chenopodium quinua (Willd.), usando energías alternativas Jiménez, J., De La Pava, S., Ochoa, J., \& Bernal Bustos, C.R. Fundación Universidad de américa. Retrieved from www.uamerica. edu.co

Foucault, A.-S., Mathé, V., Lafont, R., Even, P., Dioh, W., Veillet, S., Quignard-Boulangé, A. (2012). Quinoa extract enriched in 20-hydroxyecdysone protects mice from dietinduced obesity and modulates adipokines expression. Obesity (Silver Spring, Md.), 20(2), 2707. doi:10.1038/oby.2011.257
Gorinstein, S., Lojek, A., Pawelzik, E., Delgado-Licon, E., Medina, O. J., Moreno, M., ... Goshev, I. (2008). Comparison of composition and antioxidant capacity of some cereals and pseudocereals. International Journal of Food Science \& Technology, 43(4), 629-637. doi:10.1111/j.13652621.2007.01498.x

Graf, B. L., Poulev, A., Kuhn, P., Grace, M. H., Lila, M. A., \& Raskin, I. (2014). Quinoa seeds leach phytoecdysteroids and other compounds with anti-diabetic properties. Food Chemistry, 163, 178-85. doi:10.1016/j. foodchem.2014.04.088

Hirose, Y., Fujita, T., Ishii, T., \& Ueno, N. (2010). Antioxidative properties and flavonoid composition of Chenopodium quinoa seeds cultivated in Japan. Food Chemistry, 119(4), 1300-1306. doi:10.1016/j.foodchem.2009.09.008

Kizil, R., Irudayaraj, J., \& Seetharaman, K. (2002). Characterization of irradiated starches by using FT-Raman and FTIR spectroscopy. Journal of Agricultural and Food Chemistry, 50(14), 3912-3918. doi:10.1021/jf011652p

Koziol, M. J. (1943). Quinoa : A Potential New Oil Crop *.

Kumpun, S., Maria, A., Crouzet, S., Evrard-Todeschi, N., Girault, J.-P., \& Lafont, R. (2011). Ecdysteroids from Chenopodium quinoa Willd., an ancient Andean crop of high nutritional value. Food Chemistry, 125(4), 1226-1234. doi:10.1016/j.foodchem.2010.10.039

Lindeboom, N., Chang, P. R., Falk, K. C., \& Tyler, R. T. (2005). Characteristics of Starch from Eight Quinoa Lines. Cereal Chemistry, 82(2), 216-222.

Miranda, M., Vega-Gálvez, A., López, J., Parada, G., Sanders, M., Aranda, M., Di Scala, K. (2010). Impact of airdrying temperature on nutritional properties, total phenolic content and antioxidant capacity of quinoa seeds (Chenopodium quinoa Willd.). Industrial Crops and Products, 32(3), 258-263. doi:10.1016/j.indcrop.2010.04.019

Ng, S. C., \& Anderson, A. K. (n.d.). ISSN : 1579-4377 LIPID OXIDATION IN QUINOA ( CHENOPODIUM QUINOA ) AS DETERMINED THROUGH ACCELERATED AGING, 10101020.

Nsimba, R. Y., Kikuzaki, H., \& Konishi, Y. (2008). Ecdysteroids act as inhibitors of calf skin collagenase and oxidative stress. Journal of Biochemical and Molecular Toxicology, 22(4), 240-50. doi:10.1002/ jbt.20234

Paśko, P., Bartoń, H., Zagrodzki, P., Gorinstein, S., Folta, M., \& Zachwieja, Z. (2009). Anthocyanins, total polyphenols and antioxidant activity in amaranth and qui- 
noa seeds and sprouts during their growth. Food Chemistry, 115(3), 994-998. doi:10.1016/j. foodchem.2009.01.037

Paśko, P., Sajewicz, M., Gorinstein, S., \& Zachwieja, Z. (2008). Analysis of selected phenolic acids and flavonoids in Amaranthus cruentus and Chenopodium quinoa seeds and sprouts by HPLC. Acta Chromatographica, 20(4), 661-672. doi:10.1556/ AChrom.20.2008.4.11

Pastás, N., Pineda, Y., \& Bernal, C. (2012). Desarrollo de un polvo cosmético compacto empleando octenilsuccinato alumínico de almidón de quinua. Fundación Universidad de América. Retrieved from http://biblos.uamerica.edu.co/ cgi-bin/koha/ opac-detail.pl?biblionumber= 169974\&query_desc $=k w$, wrdl: claudio bernal
Pe, G. T., Steffolani, E., \& Leo, A. E. (2013). Study of the physicochemical and functional characterization of quinoa and kañiwa starches, 1-8. doi:10.1002/star.201200286

Prego, I., Maldonado, S., \& Otegui, M. (1998). Seed Structure and Localization of Reserves in Chenopodium quinoa. Annals of Botany, 82, 481-488.

Repo-Carrasco-Valencia, R. A., Encina, C. R., Binaghi, M. J., Greco, C. B., \& Ronayne de Ferrer, P. a. (2010). Effects of roasting and boiling of quinoa, kiwicha and kañiwa on composition and availability of minerals in vitro. Journal of the Science of Food and Agriculture, 90(12), 2068-73. doi:10.1002/jsfa.4053

Ruales, J., \& Nair, B. M. (1992). Nutritional quality of the protein in quinoa (Chenopodium quinoa, Willd) seeds.
Plant Foods for Human Nutrition, 42(1), 1-11. doi:10.1007/ BF02196067

Tang, H., Watanabe, K., \& Mitsunaga, T. (2002). Characterization of storage starches from quinoa, barley and adzuki seeds, 49, 13-22.

Taylor, S. (2009). STARCH, Chemistry and Technology. (ELSEVIER, Ed.) (Third Edit.).

Wright, K. H., Huber, K. C., Fairbanks, D. J., \& Huber, C. S. (2002). Isolation and Characterization of Atriplex hortensis and Sweet Chenopodium quinoa Starches, (C), 3-7.

Zhu, N., Kikuzaki, H., Vastano, B. C., Nakatani, N., Karwe, M. V, Rosen, R. T., \& Ho, C. (2001). Ecdysteroids of Quinoa Seeds (Chenopodium quinoa Willd .), 05, 2576-2578. 